\title{
Sleep-deprived cognitive impairment in aging mice is al- leviated by rapamycin
}

\author{
Kishore K. Mukherjee ${ }^{\mathrm{a}}$, Amanda Y. Lee ${ }^{\mathrm{a}}$, Lida Zhu ${ }^{\mathrm{a}}$, Martin Darvas ${ }^{\mathrm{b}}$, Warren Ladiges ${ }^{\mathrm{a}, *}$ \\ ${ }^{a}$ Department of Comparative Medicine, School of Medicine, University of Washington, Seattle WA 98104, USA. \\ ${ }^{b}$ Department of Pathology, School of Medicine, University of Washington, Seattle WA 98104, USA.
}

\begin{abstract}
Background: Sleep deprivation-induced cognitive impairment is a major health concern and an age-related risk factor for dementia. There is an urgent need to develop ways of preventing the adverse neurological effects of sleep deprivation, but current preclinical animal models of short-term sleep deprivation are not well described. Methods: C57BL6 mice of varying ages were sleep deprived for 4 hours a day for 4 days, and then tested with a Box maze navigation task.

Results: Sleep deprived mice at young, middle and older ages showed learning impairment that varied by strain and gender. In general, females were more sensitive to sleep deprivation than males. To determine whether sleep deprivation-induced learning impairment would respond to therapeutic intervention, an independent cohort of mice was treated with rapamycin daily during the 4 days of sleep deprivation. Mice that were sleep deprived and treated with rapamycin showed significant improvement in learning time suggesting that the cognitive impairment might be associated in part with molecular and cellular mechanisms targeted by rapamycin.

Conclusions: The observations from this study suggest that aging mice would be productive models to study pathobiology and therapeutic intervention of cognitive impairment triggered by age-related sleeping disorders in people.
\end{abstract}

Keywords: Sleep deprivation, learning impairment, aging, rapamycin

\section{Introduction}

Sleep deprivation is a major health concern in developed countries and is associated with increasing age [1-3]. The Center for Disease Control estimates that more than $35 \%$ of adults in the United States are affected by partial sleep deprivation [4-6]. This partial sleep deprivation is defined as less than seven hours of sleep, though this changes with increasing age [7-8]. Sleep disturbances such as sleep fragmentation are occurring with increasing frequency [910] and can increase the risk for age related health issues such as cancer, dementia, and heart disease. In addition,

\footnotetext{
* Corresponding author: Warren Ladiges

Mailing address: Department of Comparative Medicine, School of Medicine, University of Washington, Seattle, WA 98104, USA. Email: wladiges@uw.edu
}

Received: 18 December 2019 / Accepted: 20 December 2019 sleep deprivation is known to cause exhaustion and impaired learning ability. It has been shown that sleep deprivation impairs remote memory in aged mice, with changes in gene expression in the hippocampus [11]. We now have preliminary evidence to suggest that short term sleep deprivation in mice depletes nitric oxide in the hippocampus thereby resulting in acute vascular dysfunction [12].

Since rapamycin (Rap) has been shown to activate nitric oxide (NO) synthase in vascular endothelial cells by blocking mTOR resulting in increased NO signaling [13] there was rationale to see if Rap could prevent sleep deprived cognitive impairment. This report used a novel spatial navigation task as a readout for cognitive function in short-term sleep deprivation studies in C57BL/6 mice. Data are presented showing sleep deprived cognitive impairment occurred in aging mice and was alleviated with rapamycin treatment. These observations suggest that aging C57BL/6 mice can provide informative data on the pathophysiology and therapeutic intervention of cognitive impairment triggered by sleep deprivation as a model of age-related sleeping disorders in people. 


\section{Materials and Methods}

\section{Mice}

C57BL/6 mice were obtained from the National institute on Aging Aged Rodent Colony at Charles River, Inc. To determine age-related effects, we used 8,16 , and 22-month old mice, 10-12/cohort. Only healthy male and female mice were used. Mice were housed in a specific pathogen-free facility at the University of Washington with a standard 12:12 light-dark cycle. Water and food (LabDiet 5053) were available ad libitum. All procedures were performed as part of an approved scientific protocol in accordance with the University of Washington Institutional Animal Care and Use Committee.

\section{Sleep deprivation procedure}

This study focused on partial sleep deprivation, using a 4-hour period of sleep deprivation in contrast to more long-term studies [11]. Based on a 12:12 dark/light cycle, the animals were sleep deprived starting 4 hours after lights came on for 4 hours. Sleep deprivation was achieved through continual low stress sleep disturbances including cage tapping and gentle stroking of the back with a small brush [14-15]. Mice were subjected to 4 consecutive days of sleep deprivation 4 hours each day, followed by immediate behavior testing after the last sleepdeprivation session.

\section{Behavioral assessments}

The Box maze has been described [16]. Briefly, it consists of a rectangular clear hard-plastic box $(26.5 \mathrm{~cm}$ width, $30.5 \mathrm{~cm}$ length and $29.2 \mathrm{~cm}$ height). Each side of the box has two holes and each hole has a distinctive decoration placed above it. The holes were placed and centered 3 $\mathrm{cm}$ from the bottom of the cage. During the procedure, 7 of the holes were blocked with one escape hole open to a tube leading to an escape cage. Testing consisted of four 120 second trials. A trial was scored as completed when all four paws were inside the escape hole. The time (latency) to complete the trial was then recorded. If the mouse was unable to find the escape hole it was shown the escape hole and given a latency time of 120 seconds. Between trials, odor markers were removed from the maze with $70 \%$ ethanol.

A standardized Y-maze procedure as described previously [17] was used to assess spatial working memory. Each arm of the Y-maze was $30 \mathrm{~cm}$ long, $8 \mathrm{~cm}$ wide and 16 $\mathrm{cm}$ high. Animals were placed at the cross-section of the maze and allowed to visit the arms freely for $5 \mathrm{~min}$. Total number of arm entries and the number of arm alternations was recorded. For each alternation, we determined whether it was an alternation into a new, not previously visited arm. The total percentage of new-arm entries during the 5-min observation period was calculated for each mouse. Between animals, odor markers were removed from the maze with $70 \%$ ethanol.

\section{Rapamycin treatment}

Rapamycin (LC Laboratories, Woburn, MA) was prepared with dimethyl sulfoxide (DMSO) to make a stock concentration of $100 \mathrm{mg} / \mathrm{mL}$ and then mixed into a solution of 5\% Polyethylene Glycol, 5\% Tween 80, and 90\% Sterile $\mathrm{H}_{2} \mathrm{O}$. The dose of rapamycin was $8 \mathrm{mg} / \mathrm{kg}$ given intraperitoneally daily for 7 consecutive days. Control injections without rapamycin followed the same procedure. Mice were handled for one week prior to starting injections. Sleep deprivation began on the fourth day of treatment and ended on the seventh day when the experiment was terminated.

\section{Data analysis}

Values given in the text and in the figures are indicated as mean \pm SEM. Differences in mean values were assessed with Student's $t$-tests or appropriate repeated measures (RM) analyses of variance (ANOVAs) or ANOVA. Significant ANOVAs were followed by multiple post-hoc comparisons using Bonferroni $t$-tests. Statistical analyses were performed with Prism (GraphPad Prism, La Jolla, CA) and differences were considered significant if $P<0.05$.

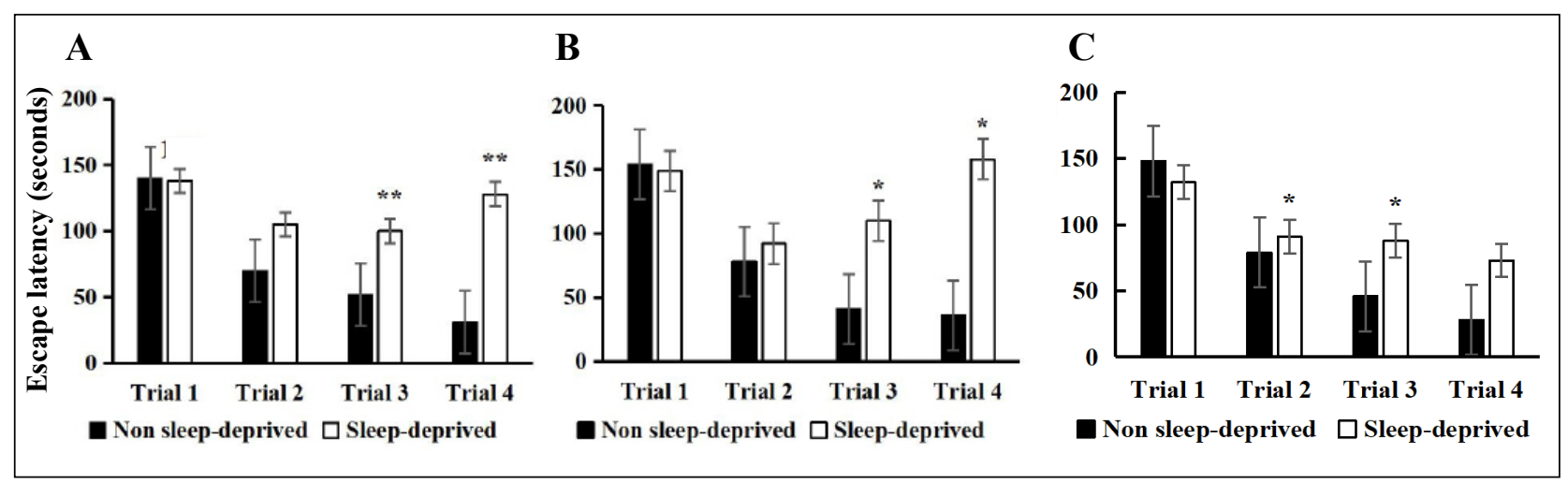

Figure 1. Sleep deprived female mice show learning impairment at young, middle and old age. (A) 8-month old females. (B) 16-month old females. (C) 22-month old females. All trials were performed on the same day and were separated by at least 2 minutes. All data are shown as averages \pm standard error of mean (SEM), 10-12/cohort. Data were analyzed by ANOVA and significant post-hoc comparisons are indicated as follows: $* P<0.05$. 


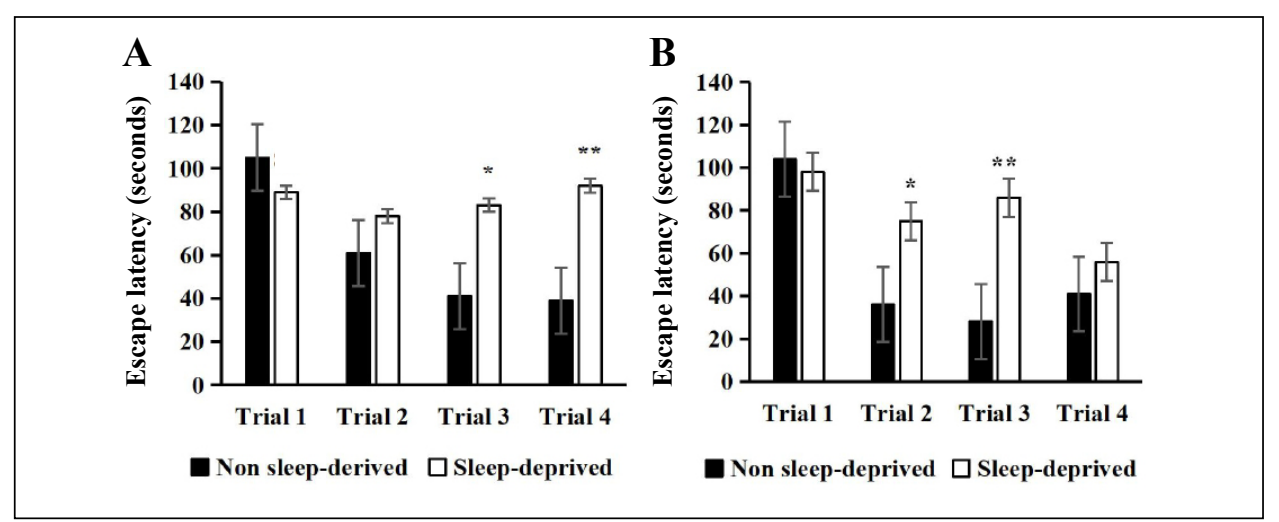

Figure 2. Sleep deprived male mice show learning impairment. (A) 8-month old males. (B) 16-month old males. All trials were performed on the same day and were separated by at least 2 minutes. All data are shown as averages \pm standard error of mean (SEM), 10-12/ cohort. Data were analyzed by ANOVA and significant posthoc comparisons are indicated as follows: $* P<0.05$ and $* * P$ $<0.01$.

\section{Results and Discussion}

\section{Sleep deprived mice show age and gender differences} in cognitive impairment.

Sleep deprivation induced escape latencies in C57BL/6 (B6) mice showed significant effects in Box maze trial number for age for females (Figure 1A. 8 months; $1 \mathrm{~B}$. 16 months; 1C. 22 months) and gender differences from males (Figure 2A. 8 months; 2B. 16 months). RM-ANOVA was used to analyze age effects on escape latencies in non-sleep deprived and sleep deprived mice. While there was no significant effect of age in non-sleep deprived or sleep deprived males (each $P>0.05$ ) and in non-sleep deprived females $(P>0.05)$, there was a statistically significant effect of age on Box-maze escape latencies in sleep deprived females (age effect $F(2,12)=6.78, P<0.05$ ). RM-ANOVA comparison of Box-maze escape latencies by gender revealed significant gender effects in 8-month old sleep-deprived mice (gender effect $F(1,23)=5.99, P$ $<0.05)$, but only a non-significant trend in 16-month old sleep-deprived mice $(P>0.05)$. The same type of analysis did not confirm significant differences between male and female non-sleep deprived mice at 8-months of age $(P>$ $0.05)$, but differences between non-sleep deprived male and female mice were significant at 16-months of age (gender effect $F(1,15)=5.61, P<0.05)$. These observations suggest that both male and female mice have sleepdeprivation induced learning impairment in the box maze, and that this deficit gets worse in female mice as they age. The study confirmed that aging mice are neurologically sensitive to short term sleep deprivation. However, a difference in severity of learning impairment between young and old mice was observed only in females. This observation is based on the Box maze learning test, so there may be other physiological features, such as other types of learning tests, memory, anxiety, and metabolic disturbances that are age sensitive. Gender differences to sleep deprivation in mice were a significant observation. The difference was not surprising since numerous studies have shown significant gender differences in people exposed to just one or two nights of sleep deprivation [18].

One aspect that we have not yet investigated is the ability to recover from short term sleep deprivation. We suspect

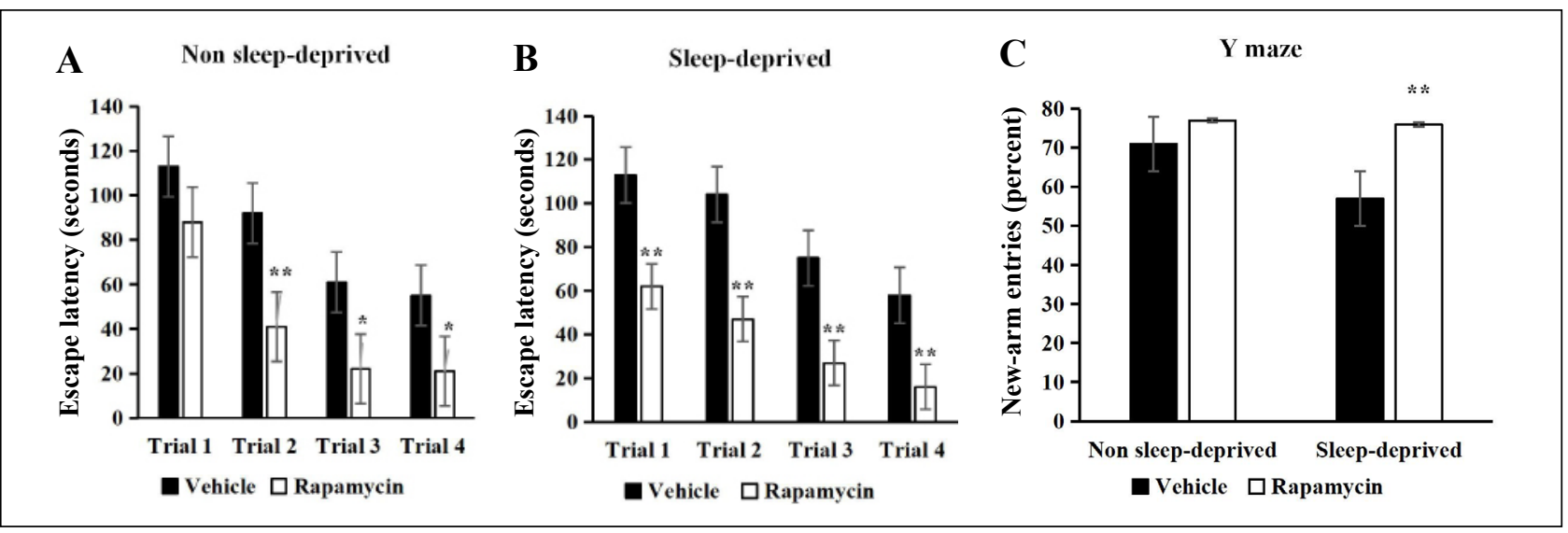

Figure 3. Rapamycin alleviates sleep deprivation induced behavioral dysfunction indicated by box-maze learning and performance in the Ymaze. (A) Box-maze escape latencies in non-sleep deprived 22-month old female mice. (B) Box-maze escape latencies in sleep-deprived 22-month old female mice. Both groups were treated with vehicle (saline) or rapamycin. Latencies were recorded during 4 trials as the time to enter the escape hole in the box maze apparatus. All trials were performed on the same day and were separated by at least 2 minutes. (C) Percentage of new-arm alterations in the Y maze by non-sleep deprived and sleep-deprived 22-month old female mice that were treated with vehicle (saline) or rapamycin. All data are shown as averages \pm SEM, 10-12/cohort. Data were analyzed by ANOVA and significant post-hoc comparisons are indicated as follows: $P<0.05$ and $* * P<0.01$. 
that older mice will take longer to recover back to homeostasis than younger mice, ie., younger mice will be more resilient to long term effects. Studies are underway to develop the Box maze for repetitive assessment over a period of time and include other behavioral tests such as the $Y$ maze. We are also looking at the metabolic repercussions that may be associated with cognitive impairment and/or adverse systemic effects.

\section{Rapamycin alleviated sleep deprived cognitive impair- ment in older mice}

We used rapamycin, a drug with demonstrated therapeutic effects on cognitive decline associated with aging in mice, to determine whether our short-term sleep deprivation model might be useful for screening therapeutic interventions. Because sleep deprivation had a stronger impact on older female mice than on male mice, we tested the effects of rapamycin on Box-maze learning in 22-month old females. Analysis of Box-maze escape latencies by vehicle- or rapamycin-treated mice with RM-ANOVA showed significant effects of trial number and treatment in non-sleep deprived and in sleep deprived mice (Figure 3). However, statistical analysis could not confirm significant differences between rapamycin-treated non-sleep deprived vs sleep-deprived mice $(P>0.05)$. The same mice were also tested with the Y Maze (24 hours after the Box-maze testing). Analysis of Y-maze new-arm entry percentages by vehicle- or rapamycin-treated mice with RM-ANOVA showed significant effects of sleep deprivation and treatment (Figure 3), but no interaction effects. Post-hoc comparisons revealed that rapamycin treatment improved the percentage of new-arm entries only in sleep-deprived mice.

Studies have shown that an increased burden of vascular pathology correlates with systemic disease and cognitive impairment [19]. Vascular impairment can result in hypoperfusion, oxidative stress, and/or inflammation, which in turn can lead to endothelial damage, infarcts, and atrophy [20]. Replenishing dysfunctional vascular cells is critical to effective repair of the vasculature. A major weapon of endothelial cells to fight vascular disease is endothelial nitric oxide synthase (eNOS), an enzyme that generates the vasoprotective molecule nitric oxide (NO). Rap has been shown to be especially effective in correcting vascular pathology associated with aging [21]. By blocking mTOR, downstream nitric oxide (NO) synthase is activated in vascular endothelial cells [13]. Vascular endothelial cells play a key role in controlling blood flow by secreting NO, which allows dilation of blood vessels to increase blood circulation.

The ability of rapamycin to prevent sleep deprived learning impairment in old mice provides preliminary evidence that targeting mTOR may be a therapeutic strategy to test these types of drugs for the prevention and treatment of sleeping disorders in the elderly. In addition, our observations suggest aging C57BL/6 mice would be excellent animal models to carry out preclinical investigations.

\section{Declarations}

Funding: This study was supported by NIA grant R01 AG057381 (Ladiges, PI).

Conflict of Interest: Warren Ladiges is a member of the Editorial Board of Aging Pathobiology and Therapeutics. All authors declare no conflict of interest and were not involved in the journal's review or desicions related to this manuscript.

\section{References}

1. Pilcher J J, Huffcutt A I. Effects of sleep deprivation on performance: a meta-analysis. Sleep, 1996, 19(4): 318326.

2. Lemons A, Saré R M, Smith C B. Chronic Sleep Deprivation in Mouse Pups by Means of Gentle Handling. JoVE (Journal of Visualized Experiments), 2018 (140): e58150.

3. Taheri S, Lin L, Austin D, et al. Short sleep duration is associated with reduced leptin, elevated ghrelin, and increased body mass index. PLoS medicine, 2004, 1(3): e62.

4. Liu Y. Prevalence of healthy sleep duration among adults-United States, 2014. MMWR. Morbidity and mortality weekly report, 2016, 65.

5. Brondel L, Romer M A, Nougues P M, et al. Acute partial sleep deprivation increases food intake in healthy men. The American journal of clinical nutrition, 2010, 91(6): 1550-1559.

6. Paruthi S, Brooks L J, D'Ambrosio C, et al. Recommended amount of sleep for pediatric populations: a consensus statement of the American Academy of Sleep Medicine. Journal of Clinical Sleep Medicine, 2016, 12(06): 785-786.

7. Cote K A, Milner C E, Osip S L, et al. Physiological arousal and attention during a week of continuous sleep restriction. Physiology \& behavior, 2008, 95(3): 353-364.

8. Tsuno N, Besset A, Ritchie K. Sleep and depression. The Journal of clinical psychiatry, 2005.

9. Gosselin D, De Koninck J, Campbell K. Novel measures to assess the effects of partial sleep deprivation on sensory, working, and permanent memory. Frontiers in psychology, 2017, 8: 1607.

10. Learning and Behavior tests https://med.stanford.edu/ sbfnl/services/bm/lm.html

11. Medic G, Wille M, Hemels M E H. Short-and long-term health consequences of sleep disruption. Nature and science of sleep, 2017, 9: 151.

12. Chikere N and Ladiges W. Acute Sleep Deprivation Induces Hepatic Oxidative Stress and Inflammation in Middleage Mice. Geropathology Research Notes,3, 2019.

13. Lin A L, Jahrling J B, Zhang W, et al. Rapamycin rescues vascular, metabolic and learning deficits in apolipoprotein E4 transgenic mice with pre-symptomatic Alzheimer's disease. Journal of Cerebral Blood Flow \& Metabolism, 2017, 37(1): 217-226.

14. De Vivo L, Nelson A B, Bellesi M, et al. Loss of sleep affects 
the ultrastructure of pyramidal neurons in the adolescent mouse frontal cortex. Sleep, 2016, 39(4): 861-874.

15. Knutson K L, Spiegel K, Penev P, et al. The metabolic consequences of sleep deprivation. Sleep medicine reviews, 2007, 11(3): 163-178.

16. Darvas M, Mukherjee K, Lee A, Ladiges W. A novel oneday learning procedure for mice. Current Protocols for Mouse Biology. 1, 2020.

17. Darvas M, Morsch M, Racz I, et al. Modulation of the $\mathrm{Ca} 2+$ conductance of nicotinic acetylcholine receptors by Lypd6. European Neuropsychopharmacology, 2009, 19(9): 670-681.

18. Ferrara M, Bottasso A, Tempesta D, et al. Gender differences in sleep deprivation effects on risk and inequality aversion: evidence from an economic experiment. PloS one, 2015, 10(3): e0120029.

19. Gorelick P B, Scuteri A, Black S E, et al. Vascular contributions to cognitive impairment and dementia: a statement for healthcare professionals from the American Heart Association/American Stroke Association. Stroke, 2011, 42(9): 2672-2713.

20. Iadecola C. The pathobiology of vascular dementia. Neuron, 2013, 80(4): 844-866.

21. Lin A L, Zheng W, Halloran J J, et al. Chronic rapamycin restores brain vascular integrity and function through NO synthase activation and improves memory in symptomatic mice modeling Alzheimer's disease. Journal of Cerebral Blood Flow \& Metabolism, 2013, 33(9): 14121421.

Cite this article as: Mukherjee $\mathrm{K}$, Lee $\mathrm{A}$, Zhu L, et al. Sleep-deprived cognitive impairment in aging mice is alleviated by rapamycin[J]. Aging Pathobiology and Therapeutics, 2019, 1(1): 05-09. 